\title{
Sensitivity analysis and metamodels for the bulk parameterization of turbulent air-sea fluxes
}

\author{
C. Pelletier, F. Lemarié, and E. Blayo \\ Univ. Grenoble Alpes, Inria, CNRS, Grenoble INP ${ }^{\dagger}$, LJK, 38000 Grenoble, France \\ + Institue of Engineering Univ. Grenoble Alpes
}

December 2017

\begin{abstract}
State-of-the-art climate models rely on bulk formulae arising from the Monin-Obukhov semiempirical theory to estimate turbulent air-sea fluxes. The mathematical structure of those formulae implies several difficulties when trying to study the numerical properties of coupling algorithms used for practical applications. This paper introduces a methodology for building physically realistic approximations of existing bulk formulae that would also satisfy suitable mathematical properties (explicit character, regularity, differentiability). This is achieved by applying the Sobol' method to compute sensitivity indices in order to reduce the number of inputs and derive a simple metamodel for the parameterization of turbulent air-sea fluxes. Numerical results show excellent agreement between our approximations and the standard bulk formulae. In particular, single column simulations using the TOGA-Coare experiment within the LMDZ atmospheric model show negligible changes in numerical results.
\end{abstract}

\section{Introduction}

\subsection{Context}

Owing to advances in computational power and in the understanding of key physical processes, most global and regional climate models routinely include a dynamical coupling between several earth system compartments (e.g., atmosphere, waves, ocean and/or sea-ice, etc). Historically confined to long-term climatic applications, coupled models are now also increasingly used for shorter seasonal forecasts or to study extreme events (e.g. tropical cyclones). Despite the level of physical refinement of these models, the representation of coupling loops remains a fundamental issue which raises several challenges of physical and numerical nature. At a physical level, existing interfacial turbulent flux parameterizations (a.k.a. bulk formulations) must be adapted to include formerly neglected processes (e.g. Bao et al., 2000; Andreas et al., 2010; Edson et al., 2013; Kudryavtsev et al., 2014). At a numerical level, the stability and the mathematical consistency of the coupling (i.e. the fact that the coupling method provides the solution to the correct coupled problem) must be carefully studied. Indeed, recent studies by Connors and Ganis (2011), Lemarié et al. (2015) or Beljaars et al. (2017) have clearly identified various numerical issues in existing practices (e.g. stability issues in atmosphere-ice coupling, lack of phasing of the diurnal cycle in air-sea coupling in climate models, etc). Those issues arise from the use of ad hoc coupling algorithms which may lead to imperfect coupling.

\footnotetext{
*Corresponding author: charles.pelletiereuniv-grenoble-alpes.fr Laboratoire LJK - Bâtiment IMAG, Université Grenoble Alpes, CS 40700, 38058 Grenoble Cedex 9, France.
} 
In this context, our final objective is to study more systematically the numerical properties of coupling algorithms used for practical applications to clarify their merits and flaws and establish more robust methods. This task is not straightforward since the continuous form of the interfacial turbulent flux parameterizations is extremely complicated and defined empirically, making it tedious to conduct thorough numerical analysis of the coupling. A first step toward our objective is thus to derive a metamodel representative of the behavior of the full parameterization but with a mathematical form that is both easy to manipulate and differentiable.

Such metamodel could also be useful to enable the differentiation of interface conditions for variational data assimilation methods or to investigate more systematically physics-dynamics coupling issues (Janiskov et al., 1999; Gross et al., 2017).

\subsection{Bulk parameterization of turbulent air-sea fluxes}

We will consider the case of the ocean-atmosphere coupling in the remaining. Note, however, that the proposed methodology can be applied to any type of coupling occurring through bulk formulae. Our understanding of the turbulence in the atmospheric surface layer is based on the Monin-Obukhov (MO) semi-empirical theory (Monin and Obukhov, 1954) which is a generalization of the classical law of the wall to stratified conditions. The MO theory predicts that the mean (in the sense of the usual Reynolds decomposition) horizontal wind $\mathbf{u}_{\mathbf{h}}=(u, v)$, potential temperature $\theta$ and humidity $q$ at a given altitude $z_{a}>0$ within the atmospheric surface layer is given by

$$
\begin{aligned}
\llbracket \mathbf{u}_{\mathbf{h}} \rrbracket_{z_{m}}^{z_{a}} & =\frac{u_{*}}{\kappa}\left\{\ln \left(z_{a} / z_{m}\right)-\psi_{m}\left(z_{a} / L\left(u_{*}, \theta_{*}, q_{*}\right)\right)\right\} \mathbf{e}_{\boldsymbol{\tau}} \\
\llbracket \theta \rrbracket_{z_{\theta}}^{z_{a}} & =\frac{\theta_{*}}{\kappa}\left\{\ln \left(z_{a} / z_{\theta}\right)-\psi_{h}\left(z_{a} / L\left(u_{*}, \theta_{*}, q_{*}\right)\right)\right\} \\
\llbracket q \rrbracket_{z_{q}}^{z_{a}} & =\frac{q_{*}}{\kappa}\left\{\ln \left(z_{a} / z_{q}\right)-\psi_{h}\left(z_{a} / L\left(u_{*}, \theta_{*}, q_{*}\right)\right)\right\}
\end{aligned}
$$

where $\llbracket X \rrbracket_{z_{1}}^{z_{2}}=X\left(z_{2}\right)-X\left(z_{1}\right)$ is the difference of a variable between two vertical levels, $z_{\text {rgh }}=$ $\left(z_{m}, z_{\theta}, z_{q}\right)$ is a set of "roughness lengths", $\psi_{m}$ and $\psi_{h}$ are so-called stability functions for momentum and scalars (e.g. Högström, 1988), $\kappa$ is the von Kármán constant, and $\mathbf{e}_{\boldsymbol{\tau}}$ is the unit vector in the direction of $\llbracket \mathbf{u}_{\mathbf{h}} \rrbracket_{z_{m}}^{z_{a}}$. In (1) the three "star variables" $\left(u_{*}, \theta_{*}, q_{*}\right)$ and the Obukhov length $L$ are turbulent scales arising from a dimensional analysis (Monin and Obukhov, 1954). The star variables are directly related to the turbulent components of ocean-atmosphere fluxes, namely the wind stress vector $\tau$, the sensible heat flux $Q_{H}$ and the latent heat flux $Q_{L}$ :

$$
\begin{aligned}
\boldsymbol{\tau} & =\rho_{a} u_{*}^{2} \mathbf{e}_{\boldsymbol{\tau}} \\
Q_{H} & =\rho_{a} c_{p} u_{*} \theta_{*} \\
Q_{L} & =\rho_{a} \Lambda u_{*} q_{*}
\end{aligned}
$$

with $\rho_{a}$ the air density in the surface layer, $c_{p}$ the specific heat of air, $\Lambda$ the latent heat of vaporization, and $\mathbf{e}_{\boldsymbol{\tau}}$ the unit vector in the direction of $\llbracket \mathbf{u}_{\mathbf{h}} \rrbracket_{z_{m}}^{z_{a}}$. The determination of $\left(u_{*}, \theta_{*}, q_{*}\right)$ requires solving (1), once an adequate closure assumption is made for $\mathbf{z}_{\mathrm{rgh}}$. Since $L$ is a function of the star variables, and the stability functions are nonlinear, this system is nonlinear and can be solved by fixed point iterations. Direct methods (e.g. Kondo, 1975; Louis, 1979) also existed prior to iterative ones, but they tend to be progressively abandoned in modern models. It is worth noting at this point that relatively large uncertainties $(20-30 \%)$ still persist within turbulent air-sea fluxes parameterizations (hereafter, bulk formulae, e.g. Brodeau et al., 2017).

In (1), several choices exist for the stability functions, which are calibrated empirically by fitting independent measurements, and several approaches are possible to estimate $z_{\text {rgh }}$, thus leading to appreciable differences between bulk formulations. Moreover, the iterative resolution of the nonlinear system 
has been rather poorly studied in terms of uniqueness of its solution and of numerical convergence (see Pagowski, 2006, for a counter example). Since earth system models are configured with increasingly higher resolution, it is expected that more and more significant departures from the neutrally-stratified case will be present in numerical simulations, potentially requiring numerous iterations to obtain the solution of (1).

\subsection{Outline of the paper}

Our objective is to present a step by step methodology to derive a direct formulation of air-sea turbulent fluxes. On top of mitigating the burden of an iterative resolution of the nonlinear system (1) and allowing an adequate treatment for asymptotics (e.g., in the case of very strong winds), the main objective is to derive a formulation that would be differentiable and make tractable the study of mathematical and numerical aspects of multiphysics coupling methods used within earth system models. It should be clear that our ambition is not to improve the physical content of current bulk formulae but to show that classical bulk formulae can be approximated by regular, explicit functions with acceptable numerical accuracy. Our approximations are not meant to be implemented within existing models; their main objective is to show that for the mathematical study of the air-sea coupled problem, considering bulk formulae as explicit, differentiable functions of physical inputs is numerically viable. Our work is similar to that of Kara et al. (2005), except that we have taken into account 3 referent bulk formulae, and built approximations that are continuous and differentiable.

The paper is organized as follows. In Section 2 the procedure to numerically solve the nonlinear system arising from the MO theory is described and three widely used bulk formulae are introduced. Then, in Section 3, we proceed to a sensitivity analysis of those three bulk formulae with respect to their inputs, to reduce the parameter space by keeping only the input variables that have been considered sufficiently influent. Thanks to this analysis based on Sobol' indices, the derivation of a metamodel is presented in Section 4 as well as numerical results with a single-column atmospheric model to illustrate that the use of a metamodel for estimating the turbulent air-sea fluxes does not significantly modify the physical results. Finally, in Section 5 we summarize our findings and conclude.

\section{Numerical evaluation of interfacial turbulent fluxes}

The determination of turbulent fluxes can be formalized as a multivariate non-linear problem which is solved via fixed point iterations. Numerical difficulties associated with this resolution are emphasized.

\subsection{Bulk formulae}

As said earlier, the turbulent components of the air-sea fluxes are directly computed from the star variables $\left(u_{*}, \theta_{*}, q_{*}\right)$ which are commonly related to transfer coefficients for momentum, heat and moisture $\left(C_{D}, C_{H}, C_{E}\right)$ by

$$
\begin{aligned}
u_{*}^{2} & =C_{D}\left\|\llbracket \mathbf{u}_{\mathbf{h}} \rrbracket_{z_{m}}^{z_{a}}\right\|\left(\llbracket \mathbf{u}_{\mathbf{h}} \rrbracket_{z_{m}}^{z_{a}} \cdot \mathbf{e}_{\boldsymbol{\tau}}\right) \\
u_{*} \theta_{*} & =C_{H}\left\|\llbracket \mathbf{u}_{\mathbf{h}} \rrbracket_{z_{\theta}}^{z_{a}}\right\| \llbracket \theta \rrbracket_{z_{\theta}}^{z_{a}} \\
u_{*} q_{*} & =C_{E}\left\|\llbracket \mathbf{u}_{\mathbf{h}} \rrbracket_{z_{q}}^{z_{a}}\right\| \llbracket q \rrbracket_{z_{q}}^{z_{a}}
\end{aligned}
$$

In theory, the terms $\llbracket X \rrbracket_{z_{X}}^{z_{a}}$ in (1) and (3) should be obtained from extrapolating each variable from the shallowest ocean level to its roughness length, which is located above the ocean surface. In practice, 
most numerical models neglect extrapolating in the ocean and consider solution profiles to be constant in the oceanic surface layer and the atmospheric viscous sublayer, therefore using solely the difference between the quantities at the height of the lowest model level in the atmosphere (typically around $10 \mathrm{~m})$ and at the depth of the shallowest model level in the ocean $(\sim 1 \mathrm{~m})$. In (3a), setting $\mathbf{e}_{\tau}=\mathbf{e}_{\llbracket \mathbf{u}_{\mathbf{h}}} \rrbracket_{z_{m}}^{z_{a}}$ yields $u_{*}^{2}=C_{D}\left\|\llbracket \mathbf{u}_{\mathbf{h}} \rrbracket_{z_{m}}^{z_{a}}\right\|^{2}$. By identification we easily get that

$$
\begin{aligned}
& C_{D}\left(z_{a}\right)=\kappa^{2}\left\{\ln \left(z_{a} / z_{m}\right)-\psi_{m}\left(\zeta\left(z_{a}\right)\right)\right\}^{-2} \\
& C_{H}\left(z_{a}\right)=\kappa \sqrt{C_{D}\left(z_{a}\right)}\left\{\ln \left(z_{a} / z_{\theta}\right)-\psi_{s}\left(\zeta\left(z_{a}\right)\right)\right\}^{-1} \\
& C_{E}\left(z_{a}\right)=\kappa \sqrt{C_{D}\left(z_{a}\right)}\left\{\ln \left(z_{a} / z_{q}\right)-\psi_{s}\left(\zeta\left(z_{a}\right)\right)\right\}^{-1}
\end{aligned}
$$

where the stability parameter $\zeta$ has been introduced

$$
\zeta\left(z_{a}\right)=\frac{z_{a}}{L\left(u_{*}, \theta_{*}, q_{*}\right)}=g \frac{\kappa z_{a}}{u_{*}^{2}}\left[\frac{\theta_{*}}{\theta_{v}\left(z_{a}\right)}+\frac{q_{*}}{q\left(z_{a}\right)+q_{0}}\right]
$$

with $\theta_{v}=\theta\left(1+q / q_{0}\right)$ the virtual potential temperature and $q_{0}$ the constant saturation humidity. $\zeta$ is a dimensionless parameter characterizing the fluid stratification due to the density gradient in a given air column: $\zeta$ and $\llbracket \theta_{v} \rrbracket_{z_{o}}^{z_{a}}$ are both 0 under neutral stratification, $\left(\zeta, \llbracket \theta_{v} \rrbracket_{z_{o}}^{z_{a}}\right)>0$ under stable stratification and $\left(\zeta, \llbracket \theta_{v} \rrbracket_{z_{0}}^{z_{a}}\right)<0$ under unstable one. Once the macroscopic variables $\llbracket u \rrbracket_{z_{o}}^{z_{a}}, \llbracket \theta \rrbracket_{z_{0}}^{z_{a}}, \llbracket q \rrbracket_{z_{0}}^{z_{a}}, \theta_{v}\left(z_{a}\right)$, and $q\left(z_{a}\right)$ are known ${ }^{1}$, an additional closure hypothesis is needed since we get six unknowns (i.e. $u_{*}, \theta_{*}$, $q_{*}$, and $z_{\text {rgh }}$ ) for only three equations (1). There are generally two strategies employed to overcome this issue:

(i) Express the roughness lengths $z_{\text {rgh }}$ in terms of $u_{*}$ (e.g. Smith, 1988; Beljaars, 1995)

(ii) Use empirically defined neutral transfer coefficients $C_{D}^{N}, C_{H}^{N}$ and $C_{E}^{N}$ (e.g. Large, 2006).

These two strategies are formally equivalent since roughness lengths and neutral transfer coefficients can directly be diagnosed from one another, by assuming neutral stratification at a fixed height $z_{N}$ :

$$
\begin{aligned}
& C_{D}\left(z_{N}\right)=C_{D}^{N}=\kappa^{2}\left\{\ln \left(z_{N} / z_{m}\right)\right\}^{-2} \\
& C_{H}\left(z_{N}\right)=C_{H}^{N}=\kappa^{2}\left\{\ln \left(z_{N} / z_{m}\right) \ln \left(z_{N} / z_{\theta}\right)\right\}^{-1} \\
& C_{E}\left(z_{N}\right)=C_{E}^{N}=\kappa^{2}\left\{\ln \left(z_{N} / z_{m}\right) \ln \left(z_{N} / z_{q}\right)\right\}^{-1}
\end{aligned}
$$

\subsection{Fixed-point problem}

All bulk formulae can be formalized as multivariable fixed point problems. Indeed, injecting (3), combined with (5), into (4) yields

$$
\begin{aligned}
{\left[u_{*} /\left\|\llbracket \mathbf{u}_{\mathbf{h}} \rrbracket_{z_{o}}^{z_{a}}\right\|\right]^{2} } & =C_{D}\left(u_{*}, \theta_{*}, q_{*}\right) \\
{\left[u_{*} \theta_{*}\right] /\left[\left\|\llbracket \mathbf{u}_{\mathbf{h}} \rrbracket z_{z_{o}}\right\| \llbracket \| \theta \rrbracket z_{z_{o}}\right] } & =C_{H}\left(u_{*}, \theta_{*}, q_{*}\right)
\end{aligned}
$$

Attempts to analytically solve (7) have not been successful yet. Therefore, the "bulk problem" is generally solved using an iterative fixed point algorithm for determining the scales $\left(u_{*}, \theta_{*}, q_{*}\right)$ that

\footnotetext{
${ }^{1}$ Throughout this study we assume that the atmospheric variables $\mathbf{u}_{h}, \theta$, and $q$ are available at a common altitude $z_{a}$, which is always the case when considering the coupling between the ocean and the atmosphere. The case where the atmospheric variables are not at the same altitude requires additional shifts in the vertical during the computation of the star variables, see Large (2006)
} 
are consistent with (7). Figure 1 displays a schematic representation of such algorithms. While their convergence has been studied numerically (see Pagowski, 2006), it has yet to be proved mathematically.



Figure 1: Bulk formulae seen as iterative algorithms. The plain line bounding box contains the bulk process in its entirety. The dashed bounding box contains the iterative loop nested within bulk formulae, $k$ denoting the iteration index; for any iterated variable $X, X_{\{k\}}$ denotes its $k^{\text {th }}$ iteration. $\left(z_{x}\right)$ is the set of roughness lengths, which are initialised by a set of constant, predefined lengths named $\left(z_{x}^{0}\right)$. For the sake of conciseness, the air moisture has been neglected; including it would keep the structure similar. Described above is a bulk algorithm relying on the roughness lengths parameterization (eg: Coare, ECMWF).

\subsection{Specificities of three different bulk formula}

Three bulk formulae will be used to numerically illustrate our approach. It should be emphasized that these bulk formulae have been developed with different constraints in mind (e.g. by prioritizing accuracy for typical conditions over the ocean rather than land surfaces, etc) and using different datasets. This translates into bulk formulae presenting non-negligible differences under certain conditions, as shown in Figure 2.

Table 1 lists some criteria by which the three considered bulk formulae differ. The NCAR-developed

\begin{tabular}{|c|c|c|c|}
\hline & CORE & Coare (3.0) & ECMWF \\
\hline gus & no & yes & yes \\
\hline$z_{0}$ & $\begin{array}{c}\text { diagnosed } \\
\text { from } C_{x}^{N} \\
\text { (see (6)) }\end{array}$ & $\begin{array}{c}\text { Smith } \\
(1988)\end{array}$ & $\begin{array}{c}\text { Smith } \\
(1988)\end{array}$ \\
\hline$C_{x}^{N}$ & $\begin{array}{c}\text { Large } \\
(2006)\end{array}$ & unnecessary & unnecessary \\
\hline$\psi_{x}$ & $\begin{array}{c}\text { Högström } \\
(1988)\end{array}$ & $\begin{array}{c}\text { Grachev } \\
\text { et al. (2000) }\end{array}$ & $\begin{array}{c}\text { Högström } \\
(1988)\end{array}$ \\
\hline
\end{tabular}

Table 1: Main differences among studied bulk formulations. "gus." stands for gustiness.

CORE formulation significantly differs from the other two formulations in that it directly fits the neutral transfer coefficient. Coare and ECMWF formulations are very similar, both using roughness-lengths following Smith (1988) and incorporating gustiness effects (Godfrey and Beljaars, 1991). This latter feature is responsible for the relative similarity between Coare and ECMWF formulations under weak 

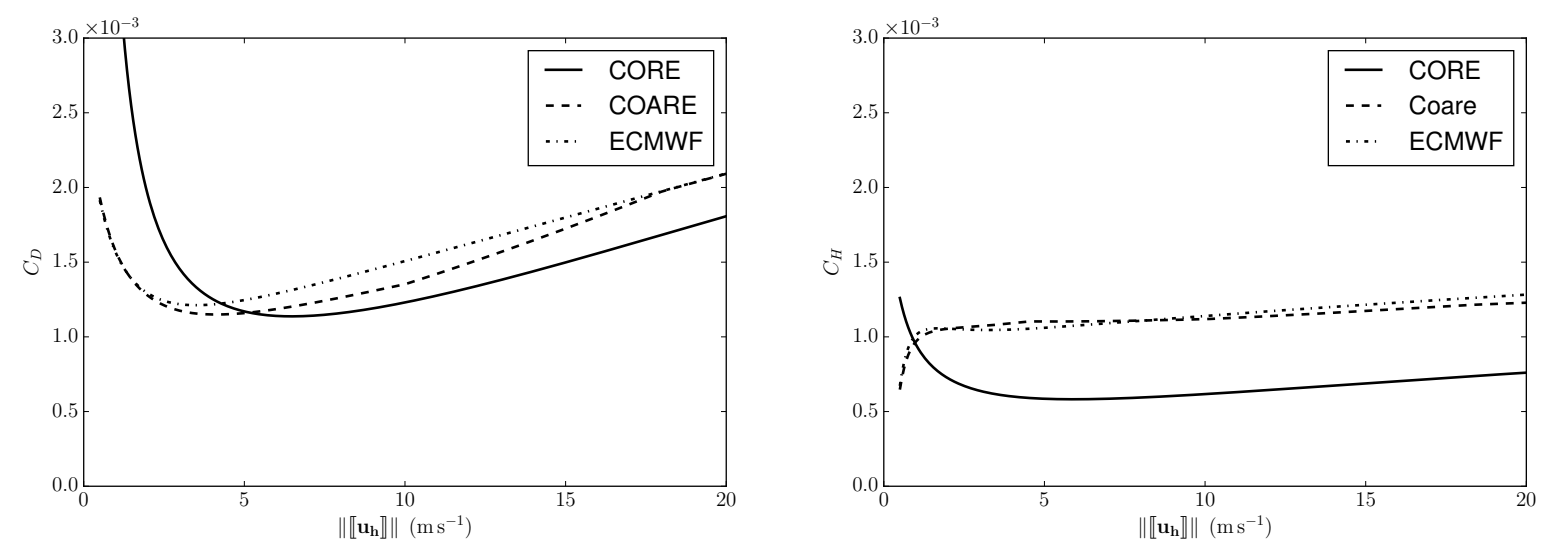

Figure 2: Comparison between different bulk algorithm output. Left panel: $C_{D}$ under unstable condition. $\llbracket \theta \rrbracket=-2 \mathrm{~K}$. Right panel: $C_{H}$ under neutral condition. $\llbracket \theta \rrbracket=0 \mathrm{~K}$.

wind configurations. For other wind configurations, the main numerical differences between Coare and ECMWF are due to their different choice of stability functions $\left(\psi_{m}, \psi_{h}\right)$. Since the ECMWF formulation is also designed to be used over land surfaces, the formulation of exchange coefficients slightly differs ${ }^{2}$ from (4) to avoid singularities when $z_{m}$ becomes large compared to $z_{a}$. However, for typical situations over the ocean this difference in the formulation of exchange coefficients is not expected to be significant.

\subsection{Numerical delicacies associated to bulk formulae}

Some numerical difficulties specific to the bulk formulae are presented. First of all, using iterative algorithms implies setting a stopping criteria. Although they tend to become rarer, there still subsists models where the iterative process is arbitrarily stopped after a constant number of iterations (typically between 2 and 5 iterations, e.g. Hourdin et al., 2006; Brodeau et al., 2017). If the maximum number of iterations is too small to converge, the turbulent fluxes thus computed may be inconsistent with MO theory. Although this tends to become less and less frequent, some numerical models still carry one single iteration; Figure 3 shows the error made on the transfer coefficients when doing so.

Second, some bulk formulae have mathematical irregularities in the sense that their outputs (most notably, $C_{H}$ and $C_{E}$ ) are discontinuous with respect to their model inputs. For instance, in the CORE algorithm, the neutral heat transfer coefficient is expressed as follows:

$$
C_{H}^{N}=\chi\left(\llbracket \theta_{v} \rrbracket_{z_{\theta}}^{z_{N}}\right) \times C_{D}^{N}
$$

where $\chi$ is a piecewise constant function, discontinuous at $\llbracket \theta_{v} \rrbracket_{z_{\theta}}^{z_{N}}=0$.

Another common practice is to impose a minimal critical value to wind speed norm to avoid a "blow up" when the wind is too low: this translates into a discontinuity in the gradient of the bulk formula with respect to this input. From a functional analysis perspective, $\mathcal{C}_{1}$-regularity of the boundary value

\footnotetext{
${ }^{2}$ To avoid singularities in logarithmic profiles it is customary for atmospheric models to replace $z_{a}$ by $z_{a}+z_{m}$ when building the non-dimensional groups in the surface-layer theory. With this modification, instead of (4a) the drag coefficient would be $C_{D}=\kappa^{2}\left\{\ln \left(\frac{z_{a}+z_{m}}{z_{m}}\right)-\psi_{m}\left(\zeta\left(z_{a}+z_{m}\right)\right)+\psi_{m}\left(\zeta\left(z_{m}\right)\right)\right\}^{-2}$, same modifications apply to $C_{H}$ and $C_{E}$.
} 


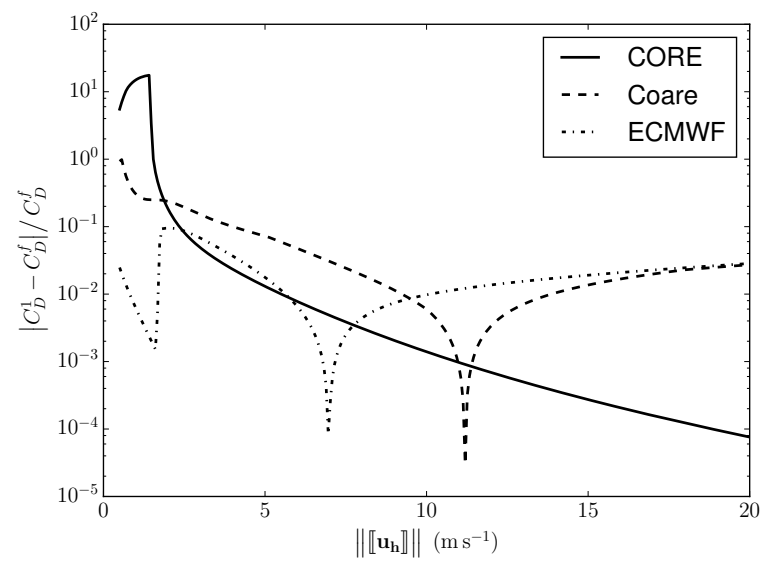

Figure 3: Relative error for $C_{D}$ computation between the first and 20th bulk iteration, where the bulks are assumed to be converged. It should be noted that the CORE bulk has an oscillatory behavior when being iterated a significant number of times, never properly converging for certain physical configurations, including the one shown above, for weak wind. Here, $\llbracket \theta \rrbracket=2 \mathrm{~K}$ and $\llbracket q \rrbracket=0$.

with respect to the problem data is a desirable property for guaranteeing mathematical well-posedness. Hence, for proper air-sea coupling, such irregularities must be avoided. At last, the implementation of bulk formulations systematically involves setting up a certain number of thresholds, for avoiding numerical "pits" where the iterative algorithm might have a divergent nature. Generally speaking, bulk formulations uncertainties increase with decreasing wind speed norm. $|\zeta| \rightarrow \infty$ corresponds to weak wind configurations; in CORE and Coare algorithms, $|\zeta|$ is bounded from above (respectively by 10 and 50) for avoiding $|\zeta| \gg 1$, which stability functions have not been tuned for.

\subsection{Assets of an explicit bulk formulae}

Hereafter, "explicit bulk formula" will refer to a bulk formula that would behave as an explicit "blackbox" functional, taking as input solely macroscopic model variables. These can be formalized as classical, mathematically explicit functions:

$$
\left(z_{a},\left\|\llbracket \mathbf{u}_{\mathbf{h}} \rrbracket_{z_{o}}^{z_{a}}\right\|, \llbracket \theta \rrbracket_{z_{o}}^{z_{a}}, \llbracket q \rrbracket_{z_{o}}^{z_{a}}, q\left(z_{a}\right), \theta\left(z_{a}\right), \ldots\right) \mapsto\left(C_{D}, C_{H}, C_{E}\right)
$$

where the input variables are yet to be determined. Using explicit approximations of bulk formulae would have several advantages:

1. Iterative bulk formulae are generally non-differentiable and may thus be responsible for the development and persistence of discontinuities in model variables (and their derivatives). A mathematically differentiable form would escape those issues.

2. An explicit bulk formulae allows to avoid a cumbersome iterative process which can be sometimes costly given the complexity of stability functions and the uncertainty on the convergence of the iterative process.

3. The proper asymptotics can be more easily enforced if they are not properly taken into account in the original bulk formulation (e.g. the Coare formulation was mostly conceived for weak wind configurations)

4. Several iterative bulk formulae could be approximated by explicit forms with the same mathematical structure (the only difference would be in the values of some coefficients) which would allow more modularity for their implementation and analysis. 
5. Simplification of the mathematical structure of bulk formulae would greatly facilitate the numerical study of coupling algorithms.

From this list, it must be clear that those arguments are primarily motivated by practical and numerical considerations. Since the physical content of the iterative bulk formulae is kept unchanged, the same amount of bias and uncertainty is to be expected in explicit ones. We will now investigate the building of such explicit numerical approximations. To do so, the first obstacle is that the bulk formulae use a lot of model input variables. The next paragraph introduces a mathematical tool that allows to reduce the number of inputs.

\section{Sensitivity analysis}

The main aim of sensitivity analysis is to determine which model inputs are the most influential on its outputs. We will apply such an analysis on the bulk formulae, formalized in the same way as (9). The first step for doing so is to determine which model inputs should be taken into consideration, which, given the complex and intertwined nature of bulk formulations, is not as obvious as it might seem. Our choice is described in Table 2.

\begin{tabular}{|c|c|c|}
\hline Name & Description & Typical range \\
\hline$z_{a}$ & first-level height & {$[1 ; 20] \mathrm{m}$} \\
\hline$\llbracket \mathbf{u}_{\mathbf{h}} \rrbracket_{z_{o}}^{z_{a}}$ & speed jump & {$[0.5 ; 25] \mathrm{m} \mathrm{s}^{-1}$} \\
\hline$\llbracket \theta_{v} \rrbracket_{z_{o}}^{z_{a}}$ & $\begin{array}{c}\text { virtual potential } \\
\text { temperature jump }\end{array}$ & {$[-10 ; 10] \mathrm{K}$} \\
\hline$\llbracket q \rrbracket_{z_{o}}^{z_{a}}$ & moisture jump & {$\left[0 ; 10^{-2}\right] \mathrm{kg} \mathrm{kg}^{-1}$} \\
\hline$\theta_{v}\left(z_{a}\right)$ & $\begin{array}{c}\text { first-level virtual } \\
\text { potential } \\
\text { temperature }\end{array}$ & {$[270 ; 320] \mathrm{K}$} \\
\hline$q\left(z_{a}\right)$ & first-level moisture & {$\left[0 ; 210^{-2}\right] \mathrm{kg} \mathrm{kg}^{-1}$} \\
\hline$P_{1}$ & first-level pressure & $\begin{array}{c}10^{5} \mathrm{~Pa} \pm \sim \\
0.1 \%\end{array}$ \\
\hline
\end{tabular}

Table 2: Choice of input variables for the sensitivity analysis of all bulk formulae.

\subsection{Sobol' indices}

We will suppose that our model has $n \in \mathbb{N}^{*}$ inputs, considered as random variables and denoted $\left(X_{i}\right)_{1 \leq i \leq n}$, and that it has one sole output, called $Y$. In our bulk formulae, $\left(X_{i}\right)_{i}$ would be the variables listed in Table 2 and $Y$ one of the three transfert coefficients, $\left(C_{x}\right)_{x \in\{D, H, E\}}$. Since standard Sobol' index (Sobol, 1993) computation methods require the independence of input variables, we will assume this hypothesis to be true. While this is not the case when extracting Table 2 variables from atmosphere models, our preliminary sensitivity analysis will be carried out using independently generated input variables. Forthcoming numerical results will show that this choice leads to efficient approximations, even within models concealing correlated variables. Moreover, there is inchoative literature on Sobol' indices with correlated inputs (e.g. Chastaing et al., 2014).

We aim at evaluating, for each $1 \leq j \leq n$, the influence of $X_{j}$ on $Y$. This is done through computing the conditional expectation $E\left(Y \mid X_{j}=x_{j}\right)$, which is the mean value of $Y$ over the distribution of $\left(X_{i}\right)_{i \neq j}$, with $X_{j}$ set to a particular value $x_{j}$. Therefore, $Z_{j}=E\left(Y \mid X_{j}\right)$ can be seen as a random variable, now considering the variations of $X_{j}$. The variance of $Z_{j}$ quantifies the influence of $X_{j}$ on the dispersion 
of $Y$, which is precisely what we are looking for. The first-order Sobol' indices are thus defined as follows:

$$
\forall j=1, \ldots, n, S_{j}^{1}=\frac{\operatorname{Var}\left(E\left(Y \mid X_{j}\right)\right)}{\operatorname{Var}(Y)}
$$

where it should be clear that $E\left(Y \mid X_{j}\right)$ is a mean computation for $Y$ over $n-1$ inputs (all besides $X_{j}$ ), and the upper variance computation is therefore done for the sole $X_{j}$ variable. The normative division by $\operatorname{Var}(Y)$ guarantees that $S_{j}^{1} \in[0 ; 1]$.

$S_{j}^{1}$ quantifies the influence of $X_{j}$ taken alone on $Y$; first-order Sobol' indices do not take into account cross-effects between input variables, meaning that, for example, all terms due to $X_{j} X_{k}(k \neq j)$ on $Y$ are not taken into account by first-order Sobol' indices. Information about these effects are contained within higher-order Sobol' indices (in the former example, by the second-order Sobol' index $S_{j, k}^{2}$ ). For each input $X_{j}$, there also exists a total Sobol' index, which is the sum of any-order Sobol' indices that include this variable. Naturally, the sum of total Sobol' indices is higher than 1, as cross-effects will be accounted for several times.

\subsection{Numerical protocol}

This paragraph aims at presenting the numerical protocol that will be used in order to carry the sensitivity analysis, which relies on the computation of Sobol' indices through the sensitivity package for $\mathrm{R}$ (Iooss et al., 2015).

The protocol is as follows:

1. Compute an estimate of the probability density function of all variable inputs from Table 2 from the ERA-Interim re-analysis (Dee et al., 2011); data from years 2000 and 2006 have been used.

2. Extract means and standard deviations from all considered variables.

3. Simulate a large number of model inputs as independent random variables, using Gaussian density functions (log-normal laws are used for positive inputs) with the previously computed means and standard deviations.

4. Compute all three bulk formulae outputs (the star variables) for each model input.

5. With the sensitivity package, using a method derived from Tissot and Prieur (2015), compute an estimate of the first and total Sobol' indices of each input variable, for each bulk formulation.

Figure 4 shows the distribution of the first two input variables, as obtained from ERA-Interim data reanalysis. Direct data from both years 2000 and 2006, which are climatologically standard in that they contain few extreme events, have been used. The dashed line density functions drawn in Figure 4 correspond to the density functions that will be used for the computing of Sobol' indices.

\subsection{Results and interpretation}

Figure 5 shows numerical results obtained on Sobol' indices. While some numerical models diagnose $q_{s}$, the sea-level air moisture, from the sea-surface temperature and the first-level pressure in order to evaluate $\llbracket q \rrbracket_{0}^{z_{a}}$, we have considered this jump to be a "raw input" for the bulk formulae. This does not impact the legitimacy of our study, as what we are interested in are the sole bulk formulae, not other numerical treatments done prior to it. 


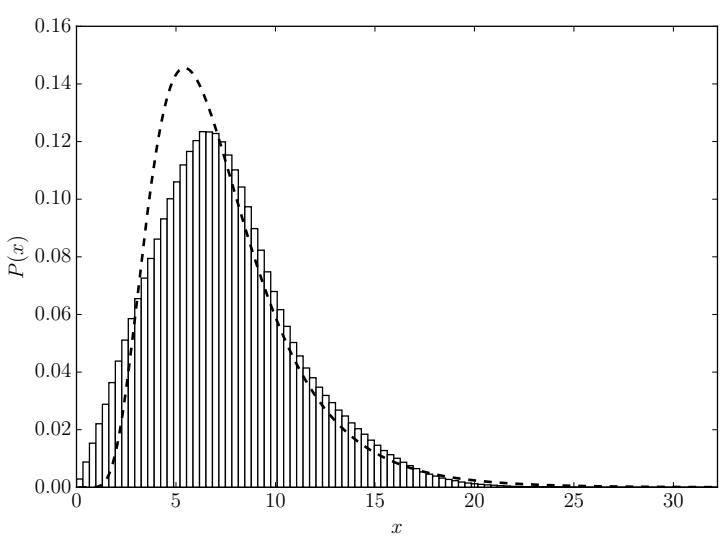

(a) $\llbracket \mathbf{u}_{\mathbf{h}} \rrbracket_{0}^{z_{a}}$

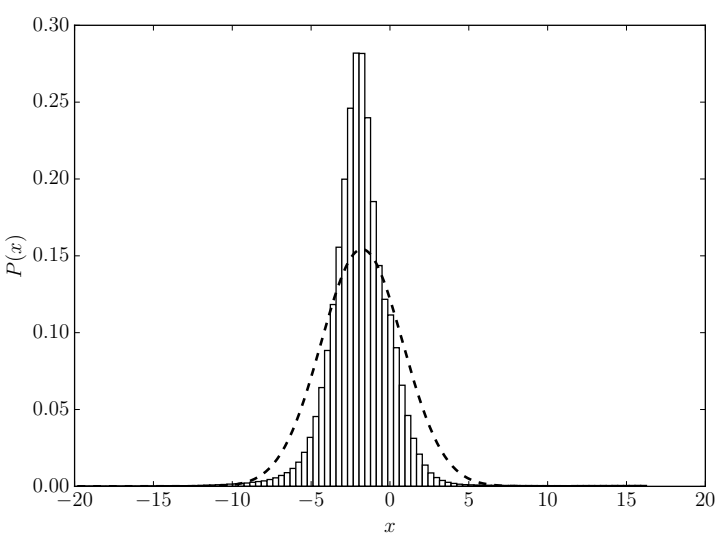

(b) $\llbracket \theta_{v} \rrbracket_{0}^{z_{a}}$

Figure 4: Normalized statistical distribution of some input variables as extracted from ERA-Interim data reanalysis (bar diagrams), and the log-normal (a) and Gaussian (b) distribution with same mean and variance.



(a)

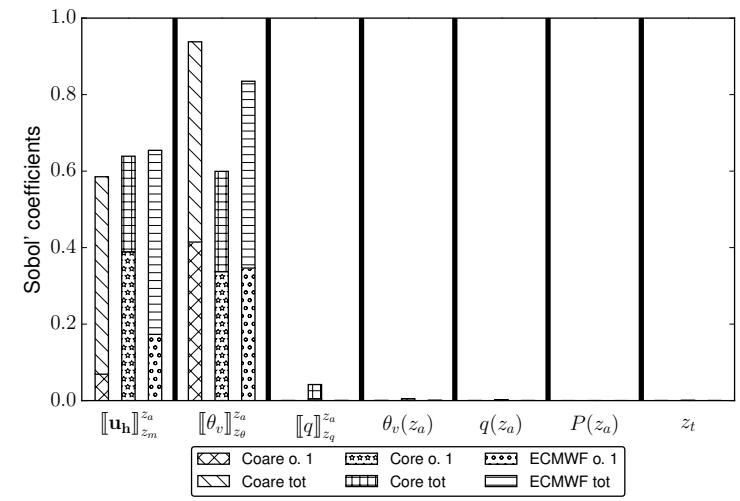

(b)

Figure 5: Sobol' indices evaluation for the 7 inputs from Table 2. (a) represents the Sobol' indices for $C_{D}$; (b), for $C_{H}$. In each diagram, each rectangle contains the Sobol' indices for one given variable. The hatch pattern distinguishes, for each input and each output, which combination of bulk (Coare, CORE or ECMWF) and index order (first or total) has been represented. For instance, the first-order Sobol' index for $\llbracket \mathbf{u}_{\mathbf{h}} \rrbracket_{z_{m}}^{z_{a}}$ in the CORE algorithm is roughly 0.65 , and its total order is roughly 0.95 . 
Results clearly show that there are two important inputs for the bulk formulae: $\llbracket \mathbf{u}_{\mathbf{h}} \rrbracket_{z_{m}}^{z_{a}}$ and $\llbracket \theta_{v} \rrbracket_{z_{\theta}}^{z_{a}}$. To some extent, $\llbracket q \rrbracket_{z_{q}}^{z_{a}}$ can also be considered as influential, yet, judging by the numerical results, its influence should be much weaker than the two other aforementionned variables. All four other input variables are much less influential. Sobol' indices for $C_{E}$ are not shown; they are very similar to $C_{H}$ (indeed, in a few bulk formulae, both these coefficients are equal).

Therefore, our explicit approximations will incorporate two input variables, taking form as such:

$$
\left(\llbracket \mathbf{u}_{\mathbf{h}} \rrbracket_{z_{m}}^{z_{a}}, \llbracket \theta_{v} \rrbracket_{z_{\theta}}^{z_{a}}\right) \mapsto \widetilde{C_{X}}, \quad X \in\{D, H, E\}
$$

It should be noted that while humidity is not shown in (11), part of its effect on transfer coefficients will be rendered through using $\theta_{v}$ instead on $\theta$. Moreover, while humidity is not explicitedly used for computing $C_{E}$, latent heat flux computation would still require $\llbracket q \rrbracket_{z_{q}}^{z_{a}}$.

\section{Technical aspects and numerical results}

Here we describe how our fit has been technically established. In particular, the asymptotic regimes are discussed as well as the numerical strategy for fitting a given bulk formulae. Finally, numerical results comparing our approximations with the standard bulk formulae are shown.

\subsection{Asymptotic regime}

As discussed earlier, proper asymptotic behavior for low wind speed regime is already built-in within iterative bulk formulae. On the other hand, the standard formulations generally do not represent the saturation of drag coefficients observed at very high winds. Such saturation has been shown using in situ data (e.g. Powell et al., 2003) and justified theoretically by Bye and Jenkins (2006). Therefore, we decided to impose this asymptotic behavior for extreme input variables and to ensure that the transition to this asymptotic regime is done in a mathematically regular way. Note that our methodology is flexible enough to implement any other type of behavior for high wind conditions since the values of exchange coefficients in this case is still matter of debate.

\subsection{Mathematical form}

As shown in figure 6, our fitting formulations are based on regionwise polynomial approximations. The regions are defined as follows:

(i) two "main" regions that account for over 93\% of possible input configurations found in the ERA-Interim reanalysis. These two regions are defined by $1.5 \leq\left\|\llbracket \mathbf{u}_{\mathbf{h}} \rrbracket_{z_{m}}^{z_{a}}\right\|\left[\mathrm{m} \mathrm{s}^{-1}\right] \leq 18$, and either $-7.5 \leq \llbracket \theta_{v} \rrbracket_{z_{\theta}}^{z_{a}} \leq-0.1$ or $0.1 \leq \llbracket \theta_{v} \rrbracket_{z_{\theta}}^{z_{a}} \leq 7.5[\mathrm{~K}]$. Unstable and stable configurations have been separated in order to achieve better numerical accuracy. In each of these two regions, the approximation is a 2-variable, $n_{d}$-degree polynomial $\left(\left(n_{d}+1\right)\left(n_{d}+2\right) / 2\right.$ degrees of freedom each), with $n_{d}$ a constant parameter to be set by the user. Sensitivity to $n_{d}$ is discussed below.

(ii) a buffer zone in between those two regions, defined as a polynomial to ensure $\mathcal{C}_{1}$ regularity at the connection, and also to ensure that the neutral case is accurately approximated.

(iii) four 1-dimensional fits for $\left\|\llbracket \mathbf{u}_{\mathbf{h}} \rrbracket_{z_{m}}^{z_{a}}\right\|=0.5 \mathrm{~m} \mathrm{~s}^{-1},\left\|\llbracket \mathbf{u}_{\mathbf{h}} \rrbracket_{z_{m}}^{z_{a}}\right\|=20 \mathrm{~m} \mathrm{~s}^{-1}, \llbracket \theta_{v} \rrbracket_{z_{\theta}}^{z_{a}}= \pm 10 \mathrm{~K}$ (solid black lines in figure 6). Those particular values define the boundary between the region where the bulk formulae are fitted and the asymptotic regime. 




Figure 6: Approximation strategy depending on the physical configurations.

(iv) a buffer zone whose main purpose is to ensure smooth transition between the main region and the 1D-fits described in (iii). For instance, weak wind configurations will be accounted for by computing $C_{X}\left(0.5 \mathrm{~m} \mathrm{~s}^{-1}, \llbracket \theta_{v} \rrbracket_{0}^{z_{a}^{1}}\right)$ and $C_{X}\left(1.5 \mathrm{~m} \mathrm{~s}^{-1}, \llbracket \theta_{v} \rrbracket_{0}^{z_{a}^{1}}\right)$, and then connecting these two values via a $\mathcal{C}^{1}$ connection over the $\left\|\llbracket \mathbf{u}_{\mathbf{h}} \rrbracket_{z_{m}}^{z_{a}^{1}}\right\|$ variable.

(v) asymptotic behaviors for input values outside of these input configurations:

- for $\left\|\llbracket \mathbf{u}_{\mathbf{h}} \rrbracket_{z_{m}}^{z_{a}}\right\|>20 \mathrm{~ms}^{-1}$, impose exponentially increasing $C_{X}$, saturating at $2.5 \times 10^{-3}$. Exponentials have been used because they can easily simulate saturation regime, however, other mathematical forms could have been chosen.

- for $\left\|\llbracket \theta_{v} \rrbracket_{z_{\theta}}^{z_{a}}\right\|>10 \mathrm{~K}$, impose $C_{X}=C_{X}( \pm 10 \mathrm{~K})$, which is consistent with the saturation of $\zeta$ that is imposed in all iterative bulk formulae.

The iterative bulk formulae of reference have been computed using the aerobul $\mathrm{k}^{3}$ package (Brodeau et al., 2017) which gathers CORE, Coare and ECMWF algorithms in a common computational framework. This package is increasingly used for ocean-only global simulations and has been treated here as a "black box".

\subsection{Numerical accuracy}

The numerical accuracy of our approximation has been tested over $0.5 \mathrm{~m} \mathrm{~s}^{-1} \leq\left\|\llbracket \mathbf{u}_{\mathbf{h}} \rrbracket_{z_{m}}^{z_{a}}\right\| \leq 20 \mathrm{~ms}^{-1}$ and $-10 \mathrm{~K} \leq \llbracket \theta_{v} \rrbracket_{z_{\theta}}^{z_{a}} \leq+10 \mathrm{~K}$. Figure 7 shows the relative error between two classical bulk algorithms (CORE and ECMWF) and their respective explicit approximations, choosing $n_{d}=6$ for the 2-variable polynomial region as described by Figure 6 .

Numerical results show that our fits have excellent accuracy over the most frequent physical configurations, which was expected by construction. Moreover, while not being as good, numerical accuracy is also satisfying over rare physical configurations. We believe this can be explained by the fact that over a 1D bounding-box, extreme values are very accurately fitted, and that the "buffer zones" (as shown

\footnotetext{
${ }^{3}$ https: / /brodeau.github.io/aerobulk/
} 


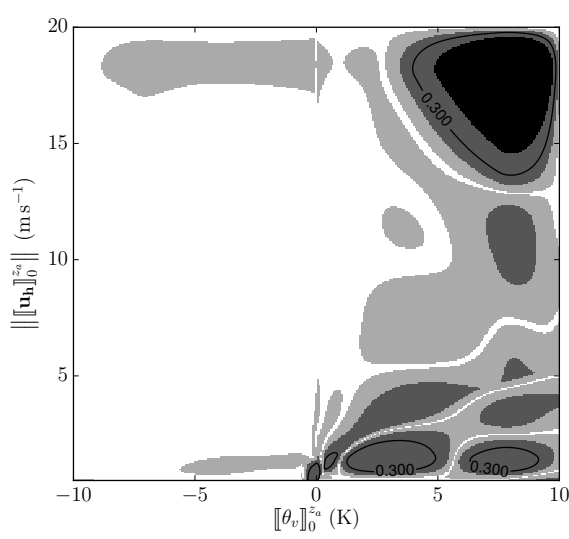

(a) $C_{D}$, ECMWF



(c) $C_{H}$, ECMWF
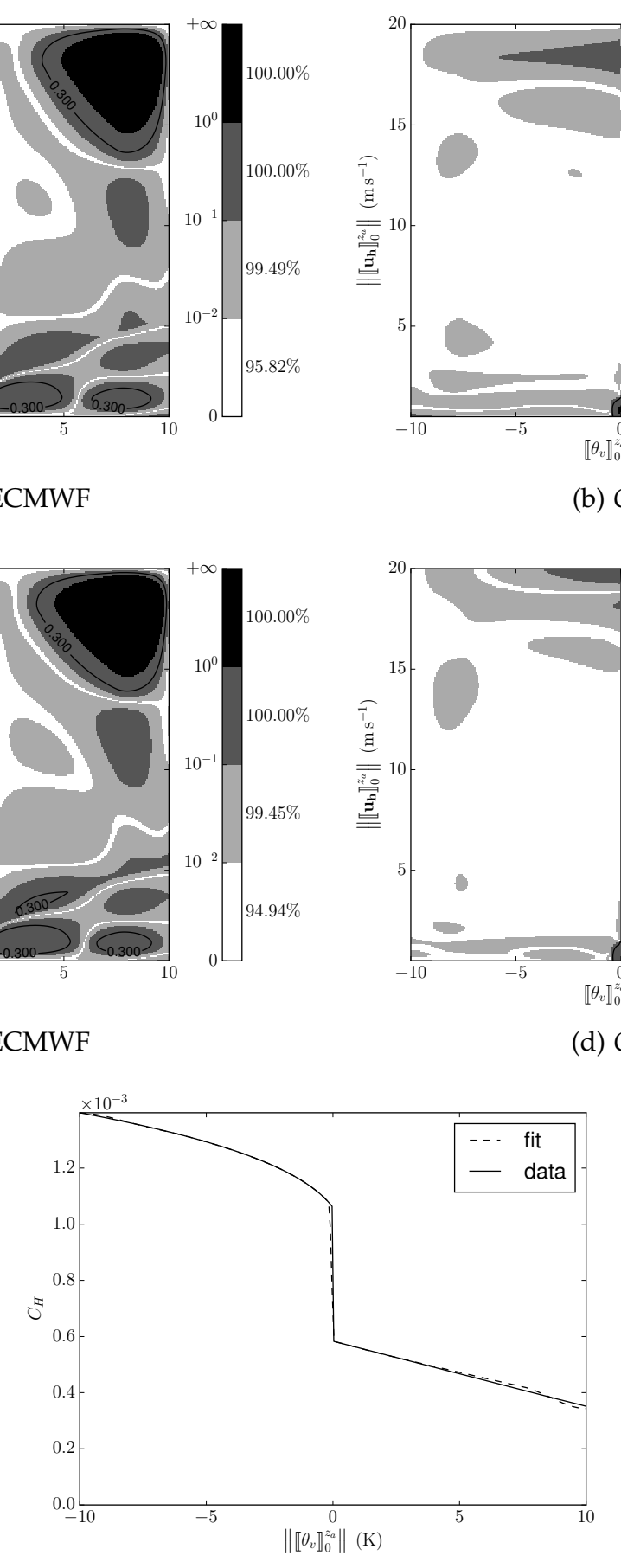

(e) $C_{H}$, CORE; (d) cut at $\left\|\llbracket \mathbf{u}_{\mathbf{h}} \rrbracket_{0}^{z_{a}}\right\|=6 \mathrm{~ms}^{-1}$.

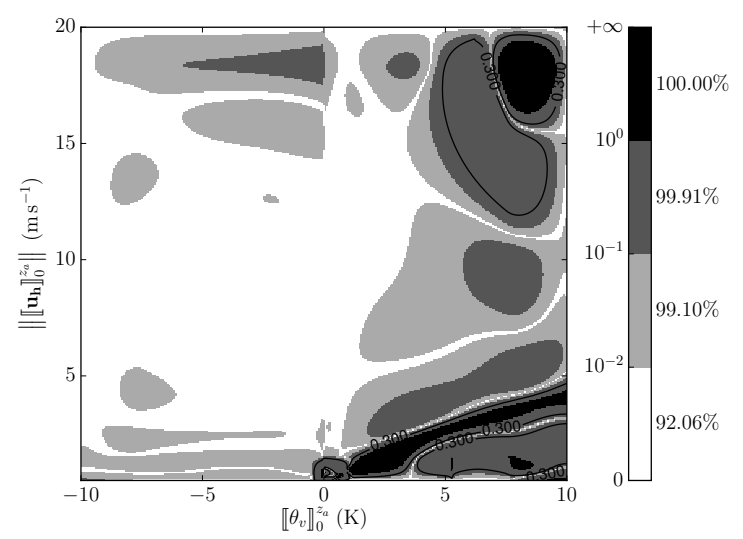

(b) $C_{D}, \mathrm{CORE}$

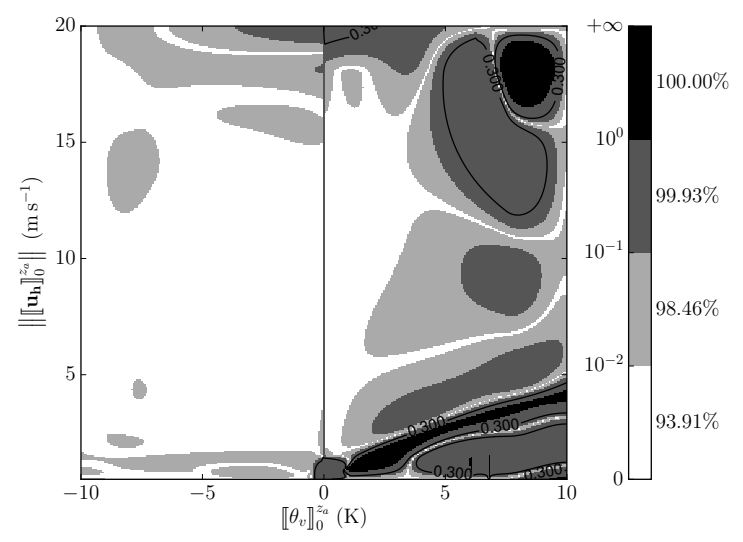

(d) $C_{H}$, CORE

Figure 7: Numerical relative error of our approximations with regards to their respective bulk formulae. The percentages given on the right hand side of the colorbar are the part of ERA-Interim bulk inputs for which the numerical output error is less than the color's upper boundary (eg: in (a), the numerical relative error on $C_{D}$ is less than $10^{-2}$ for $96.84 \%$ of the ERA Interim data inputs). The typical margin of error for bulk formulae is on the order of $30 \%$; in our fitting routines, such errors are attained for extremely rare input configurations, and small wind (especially for the CORE algorithm, which is less mathematically regular). 


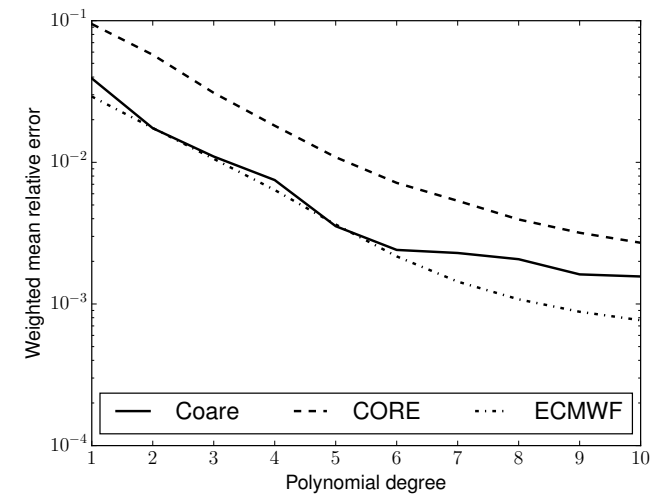

(a) $C_{D}$

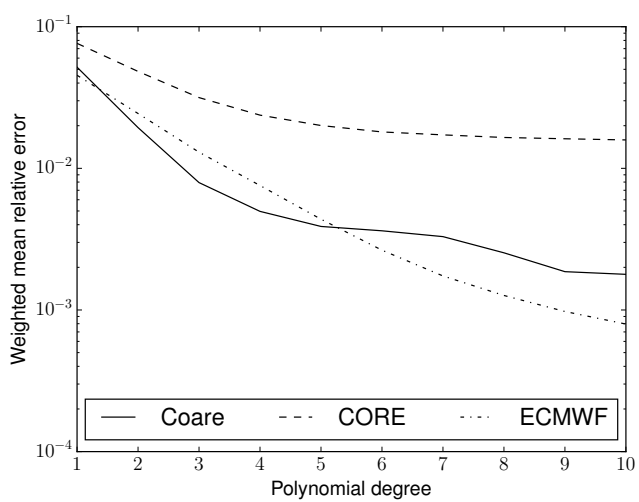

(b) $C_{H}$

Figure 8: Relative error between the classical bulk formulae and our approximations. Results have been averaged over $0.5 \mathrm{~m} \mathrm{~s}^{-1} \leq\left\|\llbracket \mathbf{u}_{\mathbf{h}} \rrbracket_{0}^{z_{a}}\right\| \leq 20 \mathrm{~ms}^{-1}$ and $-10 \mathrm{~K} \leq \llbracket \theta_{v} \rrbracket_{0}^{z_{a}} \leq+10 \mathrm{~K}$, weighted by the ERA-Interim input occurencies.

in Figure 6) tend to nudge our fit back to these values.

As expected, less accuracy is obtained for weak-wind configurations over stable stratification. Classical bulk formulations have very large uncertainties over these regions, which translate into irregularities in their mathematical formulation. Considering our explicit approximation is by nature regular, such errors are to be expected. The transition between unstable and stable configurations has large errors for the heat coefficient of the CORE algorithm (see Figure 7e). This is because we try to approximate a mathematically discontinuous function (at $\llbracket \theta_{v} \rrbracket_{z_{\theta}}^{z_{a}}=0$ ) with a regular one.

Tests have also been carried out for assessing how accurate our fitting routines are with respect to the degree of the 2-variable polynomial approximation. Figure 8 shows numerical results. As expected, the numerical accuracy increases with increasing degree. The CORE formulation, which is the least mathematically regular, has the largest error. The Coare and ECMWF formulations present similar errors, although the ECMWF one tends to show steadier increase in accuracy over large degrees. This can be explained by the complexity of stability functions in Coare which is more difficult to approximate using polynomials. Generally speaking, our numerical approximations show satisfying levels of accuracy, even for relatively small polynomial degrees. The computational cost of the 6-degree polynomial approximation corresponds to the one of 1 iteration of the CORE algorithm and of half an iteration of the Coare and ECMWF formulations (it is thus 10 times faster if we consider 5 iterations of those two algorithms).

\subsection{Numerical test: the TOGA experiment}

Our numerical approximations have been implemented within the single-column version of the LMDZ atmospheric model. We have used the default TOGA-Coare configuration of the 2014 trunk version of the 1D version of the LMDZ model (Hourdin et al., 2006), only changing the drag coefficients computation routines. The TOGA-Coare test case (Webster and Lukas, 1992) was used to test the validity of our approximations against standard bulk formulae. This test case is characterized by weak winds $\left(\left\|\llbracket \mathbf{u}_{\mathbf{h}} \rrbracket_{z_{m}}^{z_{a}}\right\| \approx 2 \mathrm{~ms}^{-1}\right)$ and unstable conditions $\left(\llbracket \theta_{v} \rrbracket_{z_{\theta}}^{z_{a}} \approx-3 \mathrm{~K}\right)$. Table 3 shows some numerical results from the LMDZ model using the non-iterative Louis (1979) formulation (the default option in LMDZ), two iterative bulk formulae of reference (Coare and CORE) and their explicit approximations 
for $n_{d}=2$ and $n_{d}=6$.

\begin{tabular}{|c|c|c|c|c|c|c|c|}
\hline \multirow{3}{*}{\multicolumn{2}{|c|}{$\begin{array}{c}\text { Reference } \\
\text { Comparison }\end{array}$}} & \multicolumn{3}{|c|}{ Coare } & \multicolumn{3}{|c|}{ CORE } \\
\hline & & \multirow{2}{*}{ LMDZ } & \multicolumn{2}{|c|}{ Fit } & \multirow{2}{*}{ LMDZ } & \multicolumn{2}{|c|}{ Fit } \\
\hline & & & $n_{d}=2$ & $n_{d}=6$ & & $n_{d}=2$ & $n_{d}=6$ \\
\hline \multirow{3}{*}{$\begin{array}{c}\delta u_{*} \\
\left(\mathrm{~ms}^{-1}\right)\end{array}$} & Mean & $1.13 e-02$ & $-9.00 e-03$ & $-7.56 e-03$ & $1.39 e-02$ & $-1.92 e-03$ & $1.90 e-03$ \\
\hline & S.D. & $5.89 e-03$ & $4.09 e-03$ & $2.63 e-03$ & $8.89 e-03$ & $5.83 e-03$ & $1.50 e-03$ \\
\hline & Max. & $2.03 e-02$ & $1.78 e-02$ & $1.44 e-02$ & $2.45 e-02$ & $1.22 e-02$ & $5.22 e-03$ \\
\hline \multirow{3}{*}{$\begin{array}{l}\delta \mathrm{T}_{1} \\
(\mathrm{~K})\end{array}$} & Mean & $7.35 e-06$ & $-2.72 e-06$ & $-8.05 e-07$ & $4.59 e-06$ & $1.58 e-06$ & $1.23 e-06$ \\
\hline & S.D. & $1.00 e-04$ & $2.46 e-05$ & $1.11 e-04$ & $1.13 e-04$ & $3.37 e-05$ & $2.94 e-05$ \\
\hline & Max. & $9.39 e-04$ & $1.61 e-04$ & $1.00 e-03$ & $1.01 e-03$ & $3.77 e-04$ & $2.17 e-04$ \\
\hline \multirow{3}{*}{$\begin{array}{c}\delta q_{1} \\
\left(\mathrm{~kg} \mathrm{~kg}^{-1}\right)\end{array}$} & Mean & $3.87 e-03$ & $-1.35 e-03$ & $3.46 e-03$ & $2.74 e-03$ & $1.95 e-03$ & $1.43 e-03$ \\
\hline & S.D. & $9.81 e-02$ & $1.23 e-02$ & $1.05 e-01$ & $1.01 e-01$ & $3.30 e-02$ & $1.80 e-02$ \\
\hline & Max. & $9.18 e-01$ & $7.98 e-02$ & $9.98 e-01$ & $9.28 e-01$ & $2.42 e-01$ & $1.37 e-01$ \\
\hline \multirow{3}{*}{$\delta \| \underset{\left(\mathrm{m} \mathrm{s}^{-1}\right)}{\left\|\mathbf{u}_{\mathbf{h}}(10 \mathrm{~m})\right\|}$} & Mean & $-8.09 e-04$ & $-1.42 e-04$ & $7.63 e-04$ & $-9.75 e-04$ & $-2.10 e-04$ & $2.46 e-05$ \\
\hline & S.D. & $1.05 e-02$ & $4.05 e-03$ & $1.06 e-02$ & $1.11 e-02$ & $6.11 e-03$ & $4.06 e-03$ \\
\hline & Max. & $1.33 e-01$ & $6.26 e-02$ & $1.31 \mathrm{e}-01$ & $1.47 e-01$ & $8.02 e-02$ & $6.14 e-02$ \\
\hline
\end{tabular}

Table 3: Statistics for the physical variables $u_{*}, T_{1}, q_{1}$ and $\left\|\mathbf{u}_{\mathbf{h}}(10 \mathrm{~m})\right\|$ during a 30-day TOGA-Coare simulation carried with the LMDZ 1D model. For each variables, we show the mean, standard deviation and maximum values of the absolute errors between a referent formulation (Coare or CORE) and either the LMDZ formulation, either our explicit approximations for $d=2$ and $d=6$.

Several remarks can be drawn from Table 3:

1. The impact of using fitted formulations instead of the referring bulk formula is at worst of the order of the uncertainty of bulk formulae itself. The difference between the outputs of a given iterative formulation and its fitted one in is smaller than the difference between the LMDZ default Louis (1979) formulation and the Coare and CORE iterative formulations.

2. While being very satisfying, the numerical accuracy displayed in Table 3 is not as good as what Figure 7 shows. We believe that this is due to the influence of the air moisture which is only taken into account via the virtual potential temperature in our approximations.

3. For physically realistic test cases, increasing the polynomial degree does not enhance accuracy as significantly as expected from the conclusions drawn from Figure 8a and Figure 8b.

\section{Conclusion and perspectives}

\subsection{Sum up}

Standard bulk formulae used in ocean-atmosphere coupled models have been introduced from a mathematical point of view in Section 2 along with critical comments on their numerical properties. This motivated the derivation of simple explicit approximations via polynomial functions based on the same physical principles as the original formulae. Section 3 describes a sensitivity analysis of the bulk problem thanks to Sobol' indices, which has led to the proper building of a simple numerical approximation in Section 4. Numerical results, showing satisfying accuracy, have been presented in two different ways: first, considering bulk algorithms as a stand-alone "black boxes" (see Figure 7), second, considering their interactions with a single column atmospheric model (see Table 3). 


\subsection{Limitations}

Aspects of the present study leave room for further improvement, as listed below.

1. Although the numerical accuracy of our approximations is globally very good for most of the physical configurations, situations for which there are significant differences between our approximations and the approximated iterative bulk formula might be of great importance when being incorporated into a GCM. However, our numerical results with the TOGA Coare test case have shown that our approximations are overall robust.

2. Recent bulk formulation developments aim at more and more accounting for sea state in general and wave characteristics in particular (see Mueller and Veron 2008, Donelan et al. 1993). While the work presented above has ignored this source of information, our methodology is flexible enough to incorporate additional input parameters. Of course, each time a new bulk formulation or a new input parameter is considered the sensitivity analysis must be repeated since the sensitivity of the outputs to the inputs can be different.

3. The choice of the physical inputs (i.e. the velocity jump and the virtual potential temperature jump) for our approximations has been done in a somewhat "non-physical" way via a sensitivity analysis. However, other more thermodynamically meaningful variables could have been considered, eg. the moist entropy potential temperature $\theta_{s}$ (Marquet, 2011). Choosing $\theta_{v}$ as an appropriate temperature scale was done for the sake of simplicity since the standard bulk formulae considered here use $\theta_{v}$ to diagnose stability. Once again, building approximations similar to those presented above for alternative bulk formulae using a different temperature scale is possible. For example, additional tests that have been carried out using $\llbracket \theta_{v} \rrbracket_{z_{\theta}}^{z_{a}} /\left\|\llbracket u_{h} \rrbracket_{z_{m}}^{z_{a}}\right\|^{2}$, which is proportional to the bulk Richardson number, as a second input (instead of $\theta_{v}$ ) gave very similar results to those presented above. However with this choice the assumptions behind the Sobol' analysis are no longer satisfied since input parameters are intrinsically correlated.

4. The LMDZ implementation of the TOGA-Coare 1D testcase used in Section 4.4 does not seem to be as sensitive to turbulent fluxes as expected. This could be explained by lateral forcing terms constraining the physics of a $1 \mathrm{D}$ column.

\subsection{Perspectives}

The FORTRAN90 routines used to derive the approximations presented in this study are available upon request. Our main motivation for building these approximations was to express complicated iterative bulk formulae with mathematically much simpler and differentiable forms. Since the physical principles behind our approximations are the same as the bulk formulae we should expect the same amount of bias and uncertainties, unless non-differentiabilities in the standard bulk formulation are more harmful than they are thought to be. On a wider perspective, we believe that having a mathematically explicit form representative of the behavior of bulk formulae is a strong requirement for the numerical study by applied mathematicians of coupling algorithms used in earth system models. Nowadays there is a serious gap between the type of coupled problems that can be thoroughly analyzed from a mathematical point of view, and the level of complexity reached by realistic coupled models including physical parameterizations. In our opinion the reduction of this gap may require the development of new mathematical tools, but more surely requires the derivation of convenient mathematical formulations mimicking the behavior of the full physical parameterizations as closely as possible.

Some aspects of the present work may also be useful for the calibration of bulk formulae with measurements. First, in current practices all available data have the same weight when trying to fit them, 
the use of PDFs as done in Section 3 could improve the quality of the fit for particular physical configurations. Second, assuming that sufficient data are available, application of the sensitivity analysis (through Sobol' indices) on turbulent flux measurements could be done. It could then be interesting to compare the Sobol' indices obtained from bulk formulations with the ones obtained from measurements. Any significant mismatch between the two would indicate that the bulk formulations do not properly represent the influence of physical inputs on the value of turbulent fluxes.

\section{Acknowledgements}

The authors would like to thank the anonymous reviewers for their valuable input, which has considerably helped improve this paper. The authors acknowledge the support of the French national research agency (ANR) through contract ANR-16-CE01-0007. F. Lemarié acknowledges the support of Mercator-Océan through PPR SIMBAD and CMEMS ALBATROS projects.

\section{References}

Andreas, E. L., Horst, T. W., Grachev, A. A., Persson, P. O. G., Fairall, C. W., Guest, P. S., and Jordan, R. E. (2010). Parametrizing turbulent exchange over summer sea ice and the marginal ice zone. Quart. J. Roy. Meteorol. Soc., 136:927-943.

Bao, J., Wilczak, J., Choi, J., and Kantha, L. (2000). Numerical simulations of air-sea interaction under high wind conditions using a coupled model: A study of hurricane development. Mon. Weather Rev., 128(7):2190-2210.

Beljaars, A., Dutra, E., Balsamo, G., and Lemarié, F. (2017). On the numerical stability of surfaceatmosphere coupling in weather and climate models. Geosc. Model Dev., 10(2):977-989.

Beljaars, A. C. M. (1995). The parametrization of surface fluxes in large-scale models under free convection. Quart. J. Roy. Meteorol. Soc., 121(522):255-270.

Brodeau, L., Barnier, B., Gulev, S. K., and Woods, C. (2017). Climatologically significant effects of some approximations in the bulk parameterizations of turbulent air-sea fluxes. J. of Phys. Oceanogr., 47(1):5-28.

Bye, J. A. and Jenkins, A. D. (2006). Drag coefficient reduction at very high wind speeds. J. of Geophys. Res., 111(C3).

Chastaing, G., Prieur, C., and Gamboa, F. (2014). Generalized Sobol sensitivity indices for dependent variables: numerical methods. J. Stat. Comp. Simu., pages 1-28.

Connors, J. and Ganis, B. (2011). Stability of algorithms for a two domain natural convection problem and observed model uncertainty. Comp. Geosc., 15(3):509-527.

Dee, D., Uppala, S., Simmons, A., Berrisford, P., Poli, P., Kobayashi, S., Andrae, U., Balmaseda, M., Balsamo, G., Bauer, P., et al. (2011). The ERA-Interim reanalysis: Configuration and performance of the data assimilation system. Quart. J. Roy. Meteorol. Soc., 137(656):553-597.

Donelan, M. A., Dobson, F. W., Smith, S. D., and Anderson, R. J. (1993). On the dependence of sea surface roughness on wave development. J. of Phys. Oceanogr., 23(9):2143-2149.

Edson, J. B., Jampana, V., Weller, R. A., Bigorre, S. P., Plueddemann, A. J., Fairall, C. W., Miller, S. D., Mahrt, L., Vickers, D., and Hersbach, H. (2013). On the exchange of momentum over the open ocean. J. of Phys. Oceanogr., 43(8):1589-1610. 
Godfrey, J. S. and Beljaars, A. C. M. (1991). On the turbulent fluxes of buoyancy, heat and moisture at the air-sea interface at low wind speeds. J. of Geoph. Res. : Oceans, 96(C12):22043-22048.

Grachev, A., Fairall, C., and Bradley, E. (2000). Convective profile constants revisited. blm, 94(3):495515.

Gross, M., Wan, H., Rasch, P. J., Caldwell, P. M., Williamson, D. L., Klocke, D., Jablonowski, C., Thatcher, D. R., Wood, N., Cullen, M., Beare, B., Willett, M., Lemarié, F., Blayo, E., Malardel, S., Termonia, P., Gassmann, A., Lauritzen, P. H., Johansen, H., Zarzycki, C. M., Sakaguchi, K., and Leung, R. (2017). Recent progress and review of Physics Dynamics Coupling in geophysical models. submitted to Reviews of Geophysics.

Högström, U. (1988). Non-dimensional wind and temperature profiles in the atmospheric surface layer: a re-evalutation. Boundary-layer Meteorol., 42:55-78.

Hourdin, F., Musat, I., Bony, S., Braconnot, P., Codron, F., Dufresne, J.-L., Fairhead, L., Filiberti, M.A., Friedlingstein, P., Grandpeix, J.-Y., Krinner, G., Levan, P., Li, Z.-X., and Lott, F. (2006). The LMDZ4 general circulation model: climate performance and sensitivity to parametrized physics with emphasis on tropical convection. Climate Dynamics, 19:3445-3482.

Iooss, B. et al. (2015). Package 'sensitivity'. Technical report, The Comprehensive R Archive Network.

Janiskov, M., Thépaut, J.-N., and Geleyn, J.-F. (1999). Simplified and regular physical parameterizations for incremental four-dimensional variational assimilation. Mon. Weather Rev., 127(1):26-45.

Kara, A. B., Hurlburt, H. E., and Wallcraft, A. J. (2005). Stability-dependent exchange coefficients for airsea fluxes. Journal of Atmospheric and Oceanic Technology, 22(7):1080-1094.

Kondo, J. (1975). Air-sea bulk transfer coefficients in diabatic conditions. Boundary-layer Meteorol., 9(1):91-112.

Kudryavtsev, V., Chapron, B., and Makin, V. (2014). Impact of wind waves on the air-sea fluxes: A coupled model. J. of Geophys. Res., 119(2):1217-1236.

Large, W. B. (2006). Surface fluxes for practitioners of global ocean data assimilation. In Chassignet, E. P. and Verron, J., editors, Ocean Weather Forecasting. An Integrated View of Oceanography, chapter 9, pages 229-270. Springer.

Lemarié, F., Blayo, E., and Debreu, L. (2015). Analysis of ocean-atmosphere coupling algorithms: Consistency and stability. Procedia Computer Science, 51(0):2066 - 2075.

Louis, J.-F. (1979). A parametric model of vertical eddy fluxes in the atmosphere. Boundary-layer Meteorol., 17(2):187-202.

Marquet, P. (2011). Definition of a moist entropy potential temperature: application to fire-i data flights. Quart. J. Roy. Meteorol. Soc., 137(656):768-791.

Monin, A. and Obukhov, A. (1954). Basic laws of turbulent mixing in the surface layer of the atmosphere. Contrib. Geophys. Inst. Acad. Sciences, USSR, 1(163):e187.

Mueller, J. A. and Veron, F. (2008). Nonlinear formulation of the bulk surface stress over breaking waves: Feedback mechanisms from air-flow separation. Boundary-layer Meteorol., 130(1):117.

Pagowski, M. (2006). An iterative solution of fluxprofile relationships in the surface layer for regional model applications. Atmosph. Env., 40(35):6892 - 6897.

Powell, M. D., Vickery, P. J., and Reinhold, T. A. (2003). Reduced drag coefficient for high wind speeds in tropical cyclones. Nature, 422(6929):279-283. 
Smith, S. D. (1988). Coefficients for sea surface wind stress, heat flux, and wind profiles as a function of wind speed and temperature. J. of Geophys. Res., 93(C12):15467-15472.

Sobol, I. M. (1993). Sensitivity estimates for nonlinear mathematical models. Math. Model. and Comp. Experiments, 1(4):407-414.

Tissot, J.-Y. and Prieur, C. (2015). A randomized orthogonal array-based procedure for the estimation of first-and second-order Sobol' indices. J. Stat. Comp. Simu., 85(7):1358-1381.

Webster, P. J. and Lukas, R. (1992). Toga coare: The coupled oceanatmosphere response experiment. Bulletin Amer. Meteorol. Soc., 73(9):1377-1416. 